\title{
Higgs Pair Production: Improved Description by Matrix Element Matching
}

\author{
Qiang Li, ${ }^{1}$ Qi-Shu Yan, ${ }^{2}$ and Xiaoran Zhao ${ }^{3}$ \\ 1 Department of Physics and State Key Laboratory of Nuclear \\ Physics and Technology, Peking University, Beijing, 100871, Chind* \\ ${ }^{2}$ College of Physics Sciences, University of Chinese Academy of Sciences, Beijing 100049, \\ China and Center for High Energy Physics, Peking University, Beijing 100871, Chind \\ ${ }^{3}$ College of Physics Sciences, University of Chinese Academy of Sciences, Beijing 100049, Chind 用
}

\begin{abstract}
Higgs pair production is crucial for measuring the Higgs boson self-coupling. The dominant channel at hadron colliders is gluon fusion via heavy-quark loops. We present the results of a fully exclusive simulation of gluon fusion Higgs pair production based on the matrix elements for $h h+0,1$ partons including full heavy-quark loop dependence, matched to a parton shower. We examine and validate this new description by comparing it with (a) Higgs Effective Theory predictions, (b) exact $h h+0$-parton sample showered by PYтнIA, and (c) exact $h h+1$-parton distributions, by looking at the most relevant kinematic distributions, such as $p_{T}^{h}, p_{T}^{h h}, M_{h h}$ spectra, and jet rate as well. We find that matched samples provide an state-of-the-art accurate exclusive description of the final state. The relevant LHE files for Higgs pair productions at the LHC can be accessed via http://hepfarm02.phy.pku.edu.cn/foswiki/CMS/HH, which can be used for relevant experimental analysis.
\end{abstract}

PACS numbers: 12.38.Cy, 12.38.-t, 13.85.Qk, 14.80.Bn

\section{INTRODUCTION}

The discovery of a 125-126 GeV Higgs-like boson [1-4], together with the early measurement on its properties [5], forsees a new era in particle physics to understand more in detail the mechanism of electroweak symmetry breaking, in which precision measurement on Higgs couplings will play a very important task, in demand of the forthcoming upgraded LHC and the promising circular or linear $e^{+} e^{-}$collider. In particular, the Higgs self coupling is crucial as it is the only portal to reconstruct and verify the Standard Model (SM) like scalar potential (see e.g. [6]):

$$
\mathcal{V}_{\mathrm{SM}}=-\mu^{2} \phi^{\dagger} \phi+\lambda\left|\phi^{\dagger} \phi\right|^{2},
$$

which leads to the triple- and quartic-Higgs couplings

$$
g_{H H H}=6 \lambda v, g_{H H H}=6 \lambda,
$$

In the SM, one has $v=\sqrt{\mu^{2} / \lambda}$ being the Higgs field vacuum expectation value, and the higgs mass $m_{H}=$ $\sqrt{2 \lambda} v$. Thus the self coupling parameter $\lambda$ is fixed by $m_{H}$, however, it may not be the case in beyond SM.

Measuring Higgs pair productions can be sensitive to $g_{H H H}$ and thus $\lambda$ (for recent phenomenology papers, see e.g. 7]). Previous Feasibility studies [8, 9] show that, for example, at the $14 \mathrm{TeV} \mathrm{LHC}$ with $3000 \mathrm{fb}^{-1}$ of data, the SM Higgs pair process can be observed with high significance and the trilinear coupling can be measured within $40 \%$ accuracy. At the LHC, Higgs boson pair production mainly proceeds via gluon fusion (GF) induced

\footnotetext{
*Electronic address: qliphy0@pku.edu.cn

${ }^{\dagger}$ Electronic address: yanqishu@gucas.ac.cn

‡Electronic address: zxrlha@gmail.com
}

by heavy-quark triangle and box loops, with the former being sensitive to the higgs trilinear couplings. In the large $M_{t}$ limit, one can integrate out the top quark filed, resulting, to a good approximation, in a simple, nonrenormalizable effective field theory (HEFT) [10],

$$
\mathcal{L}_{\text {eff }}=\frac{\alpha_{s}}{12 \pi} G_{\mu \nu}^{a} G^{\mu \nu, a} \log \left(1+\frac{H}{v}\right)
$$

with $G^{\mu \nu, a}$ being the QCD field tensor.

The leading order (LO) exact cross section has been evaluated long ago 11 13. The next-to-leading order (NLO) QCD corrections have also been calculated in Ref. [10], within the HEFT, resulting a $\mathrm{K}$ factor close to 2. The next-to-next-to-leading order (NNLO) QCD results are presented in Ref. [14, 15], again within the HEFT, finding a further increase of a factor of $\sim 1.2$ over the NLO one. The top quark mass effect at NLO has also been studied in Ref. [16], which shows a good accuracy at $\mathcal{O}(10 \%)$ can be achieved if the LO result is normalized taking into account the top quark mass.

On the other hand, in experimental analyses, it is crucial to get as precise predictions as possible for exclusive observables, such as the transverse momentum of the higgs pair, $p_{T}^{h h}$, and jet rate as well. As well known, the differential distributions predicted by HEFT is not trustable due to lost loop information, which can be easily seen if compared e.g. the HEFT $M_{h h}$ and $p_{T}^{h}$ spectrum with the exact loop results as shown in Figs. 112 One can also build in the exact loop induced matrix elements (ME) from e.g. MadLoop [17] within MadEvent [18] for showering and event generating, as done in [9]. However, beyond that, there is no available fully exclusive prediction yet so far. The reason is that one needs to compromise between the validity of HEFT and the complexity of higher loop calculations. And this is the current MonteCarlo tool status for LHC Higgs pair productions. 
It is however possible to get full exclusive control at hadron level on the complex event topology at the LHC, while still reaching approximately Next-to-leading Logarithms (NLL) accuracy, with the help of recent sophisticated matching methods between ME and parton showers (PS) [19, 20]. In PS programs, QCD radiation is generated in the collinear and soft approximation, using Markov chain techniques based on Sudakov form factors. Hard and widely separated jets are thus poorly described in this approach. On the other hand, tree-level fixed order amplitudes can provide reliable predictions in the hard region, while failing in the collinear and soft limits. To combine both descriptions and avoid double counting or gaps between samples with different jet multiplicity, an appropriate matching method is required. Several algorithms have been proposed over the years: the CKKW method, based on a shower veto and therefore on event re-weighting [19] and MLM schemes, based on event rejection [20, 21].

In this work, we report on the first matched simulation of Higgs pair production via GF in the SM that retains the full kinematic dependence on the heavy-quark loops.

The paper is organized as follows. We begin by describing our methodology. Then we present our results for the SM Higgs pair productions. We show that the matching procedure provides reliable results at the LHC and that the effects from massive quark loops can be significant. Finally we conclude in the last section.

\section{METHOD}

Our study is based on the $k_{T}$-MLM and shower$k_{T}$ matching schemes 20, 22, implemented in MadGraph/MadEvent [23], interfaced with Pythia 6.4 24] for parton shower and hadronization. As explained more in detail in our previous works for single Higgs case [25], we find it convenient to include the effects of the heavy-quark loop by simply reweighting the events generated via tree-level HEFT amplitudes.

In short, we implement di-Higgs Gluon effective interactions Eq. (3) into MAdGrapH/MADEvent, and then generate parton level events for $h h+0,1$ partons in this model. Before passing them to the PS program, events are reweigthed by the ratio of full one-loop amplitudes over the HEFT ones, $r=\left|\mathcal{M}_{\text {LOOP }}\right|^{2} /\left|\mathcal{M}_{\text {HEFT }}\right|^{2}$, where $\left|\mathcal{M}_{\text {LOOP }}\right|^{2}$ represents the full one-loop amplitude got by FeynArts 3.5 [26], FormCalc 5.3 [27] and LoopTools-2.5 package [27]. The reweighted parton-level events are unweighted, passed through PYTHIA and matched using the shower- $k_{T}$ scheme. All steps are automatic. To validate the matching procedure, the effect of changing the matching cutoff parameters such as $Q_{\min }^{\text {jet }}$ and $Q_{\min }^{\mathrm{ME}}$ in the shower- $k_{T}$ matching schemes [20, 22] on several distributions, including the $n \rightarrow n-1$ differential jet rates have been extensively assessed.

Finally, we recall that even though matrix elements

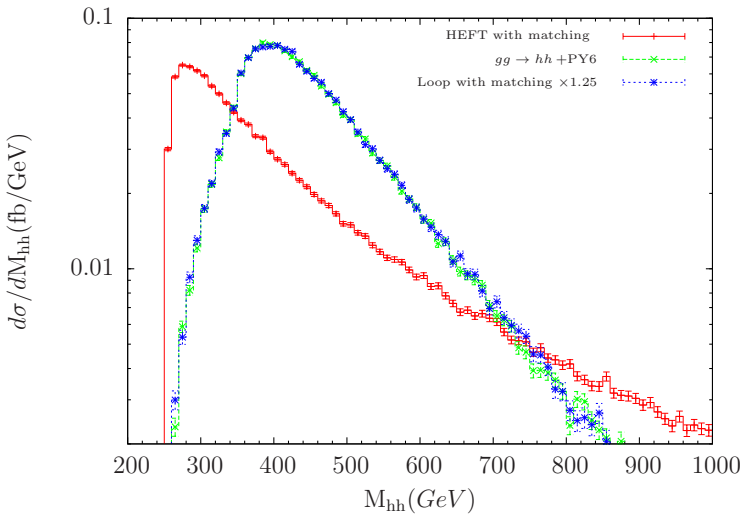

FIG. 1: $M_{h h}$ distributions in Higgs pair gluon fusion production at the $14 \mathrm{TeV}$ LHC.

for up to one final states partons are included in the simulation, the accuracy of the overall normalization of the inclusive sample is only leading order, exactly as in a purely parton-shower result.

In the following, for convenience, we call the above achieved results as Loop with matching, which will be compared with HEFT with matching, exact $h h+1$ parton level predictions from FeynArts/FormCalc/LoopTools, and exact $h h+0$ parton sample showered predictions which we get by interfacing MadLoop with our standalone MC generator for event generating and showering.

\section{SM HIGGS PAIR PRODUCTION}

To illustrate the results of our simulations for Higgs pair production via GF at the $14 \mathrm{TeV} \mathrm{LHC}$, we show a few relevant observables in Figs. 1,4, We define jets via the $k_{T}$ algorithm, with the distance measure between parton $i$ and beam $B$, or partons $i$ and $j$ as $k_{T}^{i, B} \equiv p_{T}^{i}$, $k_{T}^{i, j} \equiv \min \left(p_{T}^{i}, p_{T}^{j}\right) \sqrt{2\left(\cosh \Delta y_{i j}-\cos \Delta \phi_{i j}\right)} / D$. Here $y$ is the rapidity and $\phi$ is the azimuthal angle around the beam direction. The resolution parameter is set to $D=0.7$. Jets are required to satisfy $\left|\eta_{j}\right|<4.5$ and $p_{T}^{j}>30 \mathrm{GeV}$. For sake of simplicity, we adopt Yukawa couplings corresponding to the pole masses, i.e., for the top quark $m_{t}=173 \mathrm{GeV}$ and for the bottom-quark mass $m_{b}=4.6 \mathrm{GeV}$. Other quark masses are neglected. Throughout our calculation, we set $m_{H}=126 \mathrm{GeV}$, and adopt the CTEQ6L1 parton distribution functions (PDFs) 28] with the core process renormalization and the factorization scales $\mu_{r}=\mu_{f}$ to parton center of mass energy, $\sqrt{\hat{s}}$. For the matching performed in MADGraph/MAdEvent, as mentioned above, the shower $k_{T}$-MLM scheme is chosen, with $Q_{\min }^{\mathrm{ME}}=Q_{\min }^{\mathrm{jet}}=40 \mathrm{GeV}$.

In Figs. 1 we show Higgs pair invariant mass distribution for Standard Model Higgs pair GF production at the $14 \mathrm{TeV}$ LHC. We compare matched results in the HEFT theory and in the full theory (Loop) with Pyтнia. We 

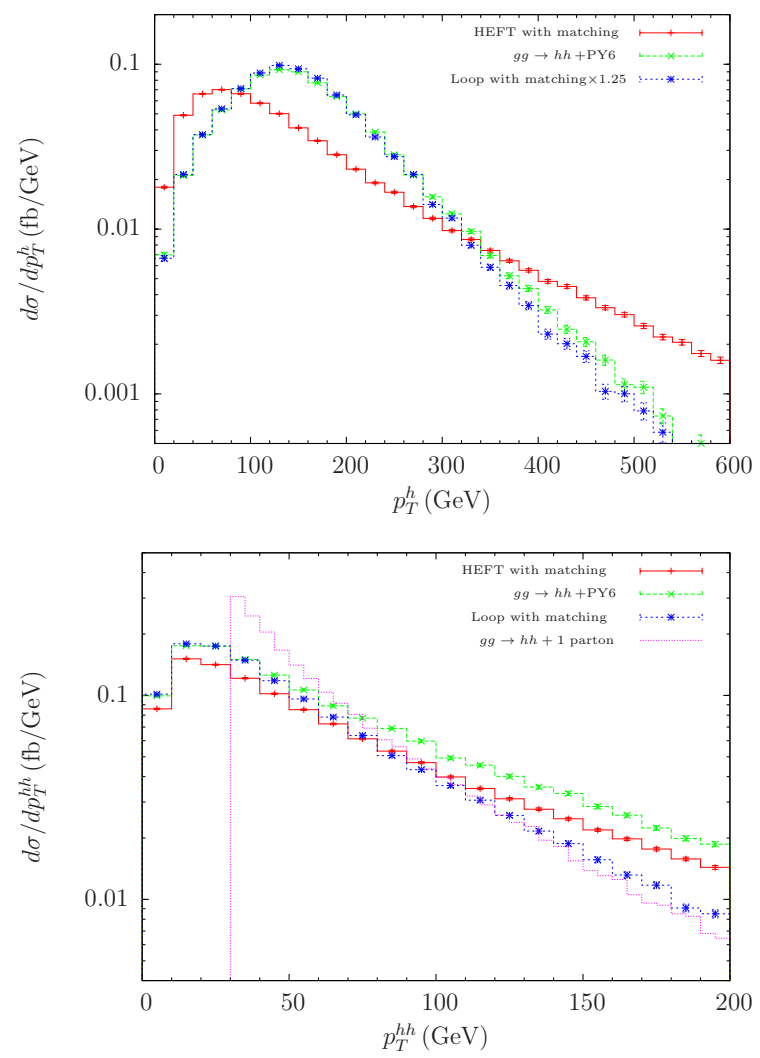

FIG. 2: Single and di-Higgs $p_{T}$ distributions for Higgs pair gluon fusion production at the $14 \mathrm{TeV}$ LHC.

also include the predictions from exact $h h+0$ parton showered with PyтнiA. The curves are all normalized to their own predictions, except the Loop with matching one which is scaled by a factor of $1.25 .{ }^{1}$ The HEFT doesn't not describe the top quark effects well as expected, while the other two agree well each other as should be.

In Figs. 2, we show single and di-Higgs $p_{T}$ distributions for Standard Model Higgs GF production at the $14 \mathrm{TeV}$ LHC. ${ }^{2}$ In the $p_{T}^{h}$ plot, one can again see the HEFT does not describe well the behavior. Instead, as expected, loop effects show a softening of the Higgs $p_{T}$, especially at quite high $p_{T}$. The Loop with matching curve agrees well with exact $h h+0$ parton showered result, except being a bit soft at high $p_{T}$ tail, which is due to the softening effect from exact $h h+1$ parton contributions included.

The $p_{T}^{h h}$ distributions essentially reflects the accompanying jet information and thus are quite sensitive to the loop effects. As one can see, the HEFT and exact $h h+0$ parton showered samples predict a too hard tail, although at low $p_{T}^{h h}$ they agree well with Loop with

\footnotetext{
1 The exact loop cross section reads $16.1 \mathrm{fb}$ and the Loop one $12.9 \mathrm{fb}$

${ }^{2}$ Note in the $p_{T}^{h h}$ plot, we don't scale the Loop with matching curve but keep its own normalization to show explicitly the agreement with exact $h h+1$ parton level curve.
}

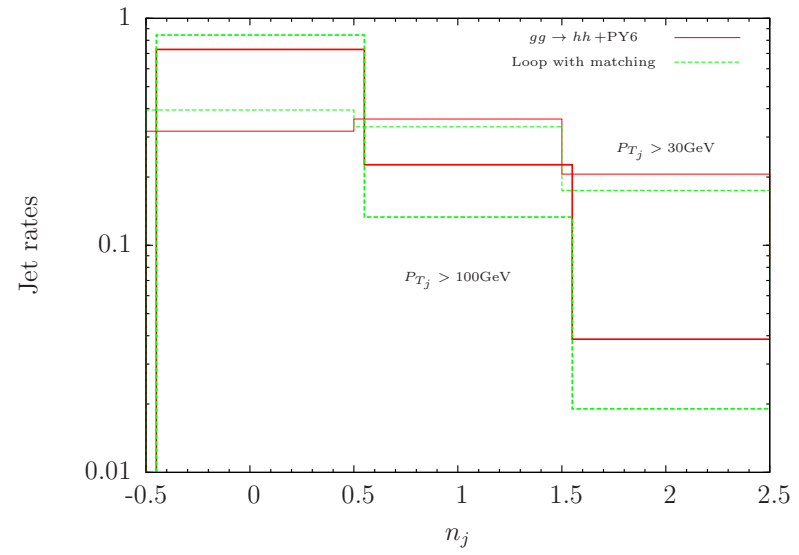

FIG. 3: Jet rates for Higgs pair gluon fusion production at the $14 \mathrm{TeV}$ LHC.
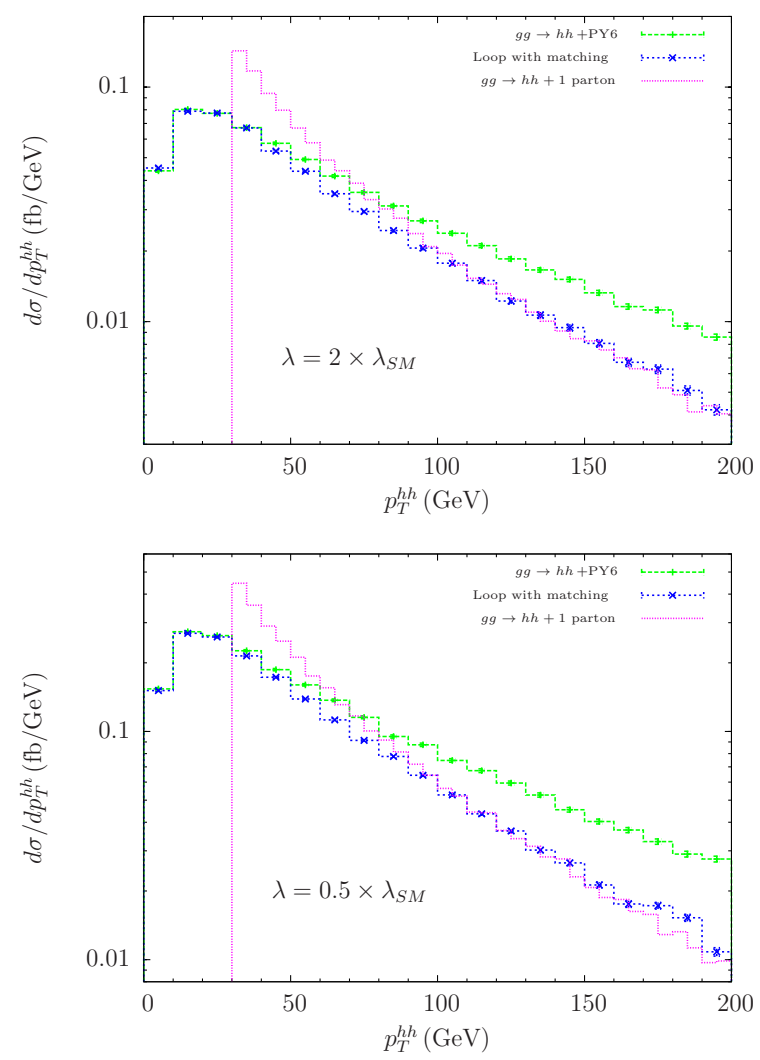

FIG. 4: $p_{T}^{h h}$ distributions for Higgs pair gluon fusion production at the $14 \mathrm{TeV}$ LHC, with the trilinear Higgs parameter $\lambda$ scaled from the SM value by a factor of 2 and 0.5 .

matching curve. This is again due to the softening effect from exact $h h+1$ parton contributions, which can be seen from the agreement between Loop with macthing and the exact $h h+1$ parton level curves. Note at very high $p_{T}^{h h}$, the Loop with matching prediction is a bit harder than the $h h+1$ one, because of additional jet radiation from shower. 
Fig. 3 shows the corresponding jet rates for different minimum jet $p_{T}^{j}$ of 30 and $100 \mathrm{GeV}$. As is readily seen from the figure, the effect of properly including loop effects is significant already at the order of $10-20 \%$ with a jet $p_{T}^{j}$ cutoff at $30 \mathrm{GeV}$, and increasingly important for larger cutoff values. This immediately translates to the effect of e.g. a jet veto with a given $p_{T}^{j}$ cutoff for the veto.

Finally, in In Fig. 4 we show the $p_{T}^{h h}$ distributions for the cases of trilinear Higgs parameter $\lambda$ scaled from the SM value by a factor of 2 and 0.5 , with similar behaviour as the above SM case, which proves our framework also works here.

\section{CONCLUSIONS}

In summary, we have presented the first fully exclusive simulation of gluon fusion inclusive Higgs pair production based on the exact one-loop matrix elements for $h h+0,1$ partons, matched to PYTHIA par- ton showers using shower- $k_{T}$ matching schemes implemented in MADGRAPH/MADEVENT. We have compared the loop reweighted matched results with the corresponding HEFT results, exact $h h+0$ parton showered results, and, when possible, with exact $h h+1$ parton level predictions. We have studied the most relevant kinematic distributions, such as Higgs pair $p_{T}$ spectra and jet rates. Our results highlight the importance of a complete loop calculation at large $p_{T}$ for a standard model Higgs. Such improved simulations might be particularly relevant in searches performed via multivariate analysis techniques where details about the kinematic distributions of the Higgs decay products and accompanying jets can have significant impact on the results.

\section{Acknowledgments}

This work is supported in part by the National Natural Science Foundation of China, under Grants No. 11175251 and 11205008.
[1] F. Gianotti, CERN Seminar, "Update on the Standard Model Higgs searches in AT- LAS", July, 4 2012. ATLASCONF-2012-093

[2] J. Incandela, CERN Seminar, "Update on the Standard Model Higgs searches in CMS", July, 42012.

[3] S. Chatrchyan et al. [CMS Collaboration], Phys. Lett. B 716, 30 (2012) [arXiv:1207.7235 [hep-ex]].

[4] G. Aad et al. [ATLAS Collaboration], Phys. Lett. B 716, 1 (2012) [arXiv:1207.7214 [hep-ex]].

[5] CMS Collaboration, CMS-PAS-HIG-13-005; ATLAS Collaboration, ATLAS-CONF-2013-034; ATLAS Collaboration, ATLAS-CONF-2013-014; CMS Collaboration, CMS-PAS-HIG13-016; Chatrchyan S, Khachatryan V, Sirunyan A M, et al[CMS Collaboration], Phys. Rev. Lett. 110, 081803 2013; ATLAS Collaboration, ATLASCONF-2013-029.

[6] S. Dawson, A. Gritsan, H. Logan, J. Qian, C. Tully, R. Van Kooten, A. Ajaib and A. Anastassov et al., arXiv:1310.8361 [hep-ex];

[7] M. J. Dolan, C. Englert, N. Greiner and M. Spannowsky, arXiv:1310.1084 [hep-ph]; J. Liu, X. P. Wang and S. -h. Zhu, arXiv:1310.3634 [hep-ph]; A. J. Barr, M. J. Dolan, C. Englert and M. Spannowsky, arXiv:1309.6318 [hep-ph]; F. Goertz, A. Papaefstathiou, L. L. Yang and J. Zurita, arXiv:1309.3805 [hep-ph]; J. Cao, Y. He, P. Wu, M. Zhang and J. Zhu, arXiv:1311.6661 [hep-ph]; R. S. Gupta, H. Rzehak and J. D. Wells, Phys. Rev. D 88, 055024 (2013) [arXiv:1305.6397 [hep-ph]]; J. Cao, Z. Heng, L. Shang, P. Wan and J. M. Yang, JHEP 1304, 134 (2013) [arXiv:1301.6437 [hep-ph]]; F. Goertz, A. Papaefstathiou, L. L. Yang and J. Zurita, JHEP 1306, 016 (2013) [arXiv:1301.3492 [hep-ph]]; D. Y. Shao, C. S. Li, H. T. Li and J. Wang, arXiv:1301.1245 [hep-ph]; U. Baur, T. Plehn and D. L. Rainwater, Phys. Rev. D 67, 033003 (2003) [hep-ph/0211224]; U. Baur, T. Plehn and
D. L. Rainwater, Phys. Rev. Lett. 89, 151801 (2002) [hep-ph/0206024]; A. Djouadi, W. Kilian, M. Muhlleitner and P. M. Zerwas, Eur. Phys. J. C 10, 45 (1999) [hep-ph/9904287]; U. Baur, T. Plehn and D. L. Rainwater, Phys. Rev. D 69, 053004 (2004) [hep-ph/0310056]; M. J. Dolan, C. Englert and M. Spannowsky, JHEP 1210, 112 (2012) [arXiv:1206.5001 [hep-ph]]; C. Han, X. Ji, L. Wu, P. Wu and J. M. Yang, arXiv:1307.3790.

[8] J. Baglio, A. Djouadi, R. Groeber, M. M. Muehlleitner, J. Quevillon and M. Spira, JHEP 1304, 151 (2013) [arXiv:1212.5581 [hep-ph]].

[9] V. Barger, L. L. Everett, C. B. Jackson and G. Shaughnessy, arXiv:1311.2931 [hep-ph].

[10] S. Dawson, S. Dittmaier and M. Spira, Phys. Rev. D 58, 115012 (1998) [hep-ph/9805244].

[11] E. W. N. Glover and J. J. van der Bij, Nucl. Phys. B 309 (1988) 282.

[12] O. J. P. Eboli, G. C. Marques, S. F. Novaes and A. A. Natale, Phys. Lett. B 197 (1987) 269.

[13] T. Plehn, M. Spira and P. M. Zerwas, Nucl. Phys. B 479, 46 (1996) [Erratum-ibid. B 531, 655 (1998)] [hep$\mathrm{ph} / 9603205]$.

[14] D. de Florian and J. Mazzitelli, Phys. Lett. B 724 (2013) 306 [arXiv:1305.5206 [hep-ph]]; D. de Florian and J. Mazzitelli, JHEP 1212 (2012) 088 [arXiv:1209.0673 [hep-ph]].

[15] D. de Florian and J. Mazzitelli, Phys. Rev. Lett. 111, 201801 (2013) [Phys. Rev. Lett. 111, 201801 (2013)] [arXiv:1309.6594 [hep-ph]].

[16] J. Grigo, J. Hoff, K. Melnikov and M. Steinhauser, arXiv:1305.7340 [hep-ph].

[17] V. Hirschi, R. Frederix, S. Frixione, M. V. Garzelli, F. Maltoni, et al., "Automation of one-loop QCD corrections," JHEP, vol. 1105, p. 044, 2011.

[18] F. Maltoni and T. Stelzer, JHEP 0302, 027 (2003) [hep$\mathrm{ph} / 0208156]$. 
[19] S. Catani, F. Krauss, R. Kuhn, and B. Webber, "QCD matrix elements + parton showers," JHEP, vol. 0111, p. 063, 2001.

[20] J. Alwall, S. Hoche, F. Krauss, N. Lavesson, L. Lonnblad, et al., "Comparative study of various algorithms for the merging of parton showers and matrix elements in hadronic collisions," Eur.Phys.J., vol. C53, pp. 473-500, 2008.

[21] M. L. Mangano, M. Moretti, F. Piccinini, and M. Treccani, "Matching matrix elements and shower evolution for top- quark production in hadronic collisions," JHEP, vol. 01, p. 013, 2007.

[22] J. Alwall, S. de Visscher, and F. Maltoni, "QCD radiation in the production of heavy colored particles at the LHC," JHEP, vol. 0902, p. 017, 2009.

[23] J. Alwall et al., "MadGraph/MadEvent v4: The New
Web Generation," JHEP, vol. 0709, p. 028, 2007.

[24] T. Sjostrand, S. Mrenna, and P. Z. Skands, "PYTHIA 6.4 Physics and Manual," JHEP, vol. 0605, p. 026, 2006.

[25] J. Alwall, Q. Li and F. Maltoni, Phys. Rev. D 85, 014031 (2012) [arXiv:1110.1728 [hep-ph]].

[26] T. Hahn, "Generating Feynman diagrams and amplitudes with FeynArts 3," Comput.Phys.Commun., vol. 140, pp. 418-431, 2001.

[27] T. Hahn and M. Perez-Victoria, "Automatized one loop calculations in four-dimensions and D-dimensions," Comput.Phys.Commun., vol. 118, pp. 153-165, 1999.

[28] J. H. J. Pumplin, D. R. Stump et al., "New generation of parton distributions with uncertainties from global QCD analysis," JHEP, vol. 0207, p. 012, 2002. 